\title{
CALIDAD, SISTEMA DE SALUD Y GOBERNANZA. EL CASO ARGENTINO
}

\begin{abstract}
Sergio G. Litewka*
Resumen: Argentina ha sido un campo fértil para los intentos de reforma del sector salud, en la búsqueda por mejorar la calidad de sus servicios y, consecuentemente, la accesibilidad y la equidad del mismo. Los resultados obtenidos no han sido proporcionales a los esfuerzos desarrollados. Aunque la bioética debería, como forma de reflexión interdisciplinaria, participar en la fundamentación de nuevas políticas sanitarias y sus efectos sobre los usuarios, parece haber permanecido ajena, en general, a los graves problemas derivados de la corrupción, prefiriendo enfocarse en cuestiones vinculadas a planteos abstractos de justicia y solidaridad, atribuyendo los fracasos a la imposición de modelos económicos foráneos.
\end{abstract}

Palabras clave: Argentina, corrupción, salud, ética

\section{QUALITY, HEALTH SYSTEM AND GOVERNANCE. THE ARGENTINEAN CASE}

\begin{abstract}
Argentina had been a fertile ground for reform attempts, focusing in the quality of its health services and subsequently, improving the accessibility and equity to the system. Even though bioethics, as a tool for interdisciplinary reflection, should had been a participant for the foundations of health policies and the impact on its users, it seems that local bioethics had omitted the deleterious consequences created by the lack of governance and corruption on the Argentinean public and social security healthcare systems, choosing instead for focusing in blurry discussions about universal issues related to solidarity and justice, blaming for the failures to the imposition of foreign economical models.
\end{abstract}

Key words: Argentine, corruption, health, ethics

\section{QUALIDADE, SISTEMA DE SAÚDE E GOVERNANÇA. O CASO ARGENTINO}

Resumo: Argentina tem sido um campo fértil para os intentos de reforma do setor saúde, na busca pela melhoria da qualidade de seus serviços e, consequentemente, melhoria de sua acessibilidade e equidade. Os resultados obtidos não têm sido proporcionais aos esforços desenvolvidos. Ainda que a bioética devesse, como forma de reflexão interdisciplinar, participar na fundamentação de novas políticas sanitárias e de seus efeitos sobre os usuários, estas parecem ter permanecido alheias, em geral, aos graves problemas derivados da corrupção, preferindo-se enfocar questôes vinculadas a propostas abstratas de justiça e solidariedade, atribuindo os fracassos à imposição de modelos econômicos estrangeiros.

Palavras-chave: Argentina, corrupção, saúde, ética

\footnotetext{
Profesor Investigador Asistente, Programas de Ética de la Universidad de Miami, Estados Unidos Correspondencia: slitewka@med.miami.edu
} 


\section{Introducción}

La Declaración de Alma Ata sobre Salud para Todos en el año 2000, enunciada en 1978, planteaba objetivos ambiciosos en la búsqueda de mejorar la accesibilidad a los sistemas de salud. Siendo que la meta parecía estar suficientemente lejana, brindaba la oportunidad de abrigar esperanzas para quienes deseaban que la equidad en el terreno de la salud pública fuera una realidad(1).

Salud para Todos en el año 2000 enfatizaba la necesidad de morigerar las injusticias resultantes de la distribución irregular de los recursos para la salud, producidas por las diferencias entre países "pobres" y países "ricos". En su punto décimo, Alma Ata llamaba la atención sobre el despilfarro originado por las inversiones con fines bélicos, exhortando a que estos fondos se dirigieran, en cambio, hacia acciones de atención médica primaria y de mejora en la calidad de los sistemas de salud.

Durante los años subsiguientes, Alma Ata fue el mantra utilizado por muchos sanitaristas, especialmente aquellos que representaban los intereses de las naciones consideradas "en vías de desarrollo". Indudablemente, la Declaración era atractiva y no podía sino simpatizarse con la expectativa de "promover (...) el máximo compromiso, a nivel individual y comunitario, en el planeamiento, organización, operación y control del cuidado primario de la salud, haciendo pleno uso de los recursos locales, nacionales y otros disponibles, y a través de estos conseguir mediante la educación apropiada y la capacidad de las comunidades de mejorar la provisión de los servicios ofrecidos".

Años antes, en 1966, Avedis Donabedian lanzaba su clásico modelo para estudiar y mejorar la calidad de los servicios de las organizaciones de salud basado en estructura, proceso y resultado(2).

Otros autores coincidían en el intento por incorporar paradigmas propios de la industria (Juran, Deming) a los sistemas de salud, con la convicción de que una mejor comprensión por parte de las organizaciones acerca de cómo mejorar la calidad de sus prestaciones repercutiría positivamente sobre los beneficiarios de las mismas. Básicamente, la idea central seguía siendo la consecución de objetivos de justicia distributiva, promoviendo la igualdad, mediante la utilización racional y eficaz de los recursos disponibles. Nada de esto estaba lejos de los postulados de Alma Ata.
En la misma tónica, durante la década del 90 los organismos multilaterales de crédito y otras agencias internacionales comenzaron a apoyar en forma entusiasta lo que sería conocido como "procesos de reforma en el sector de la salud". Casi la totalidad de los países de América Latina participaron en estas actividades. Había coincidencia en un conjunto de componentes básicos, a saber: mejoras en la gestión, en los modelos económico-financieros y en el manejo de los recursos humanos, y capacitación del personal sanitario e implantación de criterios de calidad en la producción de los servicios de salud. El criterio predominante era que, apoyando la formulación de mecanismos de gerencia adecuados, dentro del contexto de criterios de calidad, sería posible atenuar las groseras inequidades existentes como consecuencia de las brechas sociales en naciones con históricas disparidades socioeconómicas.

A pesar de esta declaración de buena voluntad, en la mayoría de los países involucrados las reformas apenas quedaron reflejadas en los informes preparados por las empresas consultoras y los documentos archivados en las agencias que intervinieron. En la práctica, la imposibilidad de modificar el statu quo de las organizaciones y la resistencia de los grupos tradicionales de poder dentro de los modelos médico-hegemónicos -característicos en América Latina- contribuyeron a que el año 2000 encontrara a muchas de estas naciones en condiciones similares a las existentes al inicio de los intentos de reforma. Así, salud para todos seguiría siendo una declamación aceptada por todos, pero huérfana de herramientas que posibilitaran plasmarla en el mundo real.

La difusión de la bioética como disciplina en los países desarrollados, si bien no tiene relación directa, es relativamente contemporánea a la Declaración de Alma Ata.

Rodríguez Yunta, analizando la bioética latinoamericana, sostiene que "(la bioética) se ha establecido progresivamente como una plataforma común sobre la cual expertos de diversas disciplinas -como medicina, filosofía, derecho, teología, antropología y ciencias sociales- colaboran para resolver problemas en el ámbito de la salud y la relación con la nueva tecnología aplicada a la medicina y la ciencia"(3). De tal forma, la bioética no sólo es una disciplina que se ocupa de las relaciones entre médicos y pacientes o entre investigadores y sujetos, sino que su mirada se extiende a cuestiones vinculadas con la salud pública, políticas 
ambientales y sociales, así como a las consecuencias sanitarias derivadas de la exclusión social.

A pesar de esto, esta disciplina no fue considerada al formular el marco teórico que daría lugar al entramado de los procesos de reforma en la región latinoamericana. Si las mejoras buscaban impactar favorablemente en la equidad y el acceso a los sistemas de salud, favoreciendo la participación comunitaria, la transparencia y, en última instancia, mejorando la calidad del sistema mediante procesos de acreditación, certificación y subsidiariedad, podría argumentarse que, como herramienta transdisciplinaria, hubiera permitido una aproximación holística al problema. Tal vez en ese momento la bioética no estaba demasiado afianzada como para estimular su participación o, tal vez, al momento en América Latina, estaba demasiado ocupada en los fenómenos individuales, sin posibilidad de atender a los cambios que se preparaban para el conjunto social.

En efecto, en cualquier proceso de reforma en el sector de la salud las preocupaciones centrales se basan en los problemas de costos y las dificultades en el acceso. No importa la ideología que prevalezca, los factores que se consideran están relacionados con recursos siempre limitados en relación con la magnitud de la demanda (esto independientemente de lo desarrollada o carente que sea una sociedad), por lo que se necesitaba establecer políticas de contención, determinando prioridades que, al menos en teoría, deberían sustentarse en principios de justicia y equidad.

Va de suyo que la bioética no reemplaza al conocimiento del economista o del administrador de los servicios de salud. Es más bien complementaria pero insoslayable, cuando lo que está en juego son decisiones que tendrán impacto sobre la población de usuarios de los sistemas que reciben finaciamiento público (sea éste originado en el Estado o dentro del sistema de la seguridad social).

Cabe preguntarse si la bioética, como disciplina ya activa en los 90, fue ignorada porque los planificadores desconocían de su existencia o porque se consideró que era incapaz de plantear soluciones racionales y aplicables a los complejos problemas tratados.

\section{Calidad en salud y ambiente ético: el caso argentino}

Pocos países de la región recibieron tantos recursos durante los ańos 90, con el objeto de mejorar la calidad de sus sistemas públicos de atención médica, como Argentina. En efecto, en 1995, a través del Programa de Reforma del Sector Salud (PRESSAL), se le otorgó al país un crédito de US\$ 101 millones, con el objeto de favorecer la reconversión de todo el sistema público de salud. Esto incluía tanto a un conjunto de hospitales dependientes del Estado como a un sector de la seguridad social (obras sociales) financiado por las asociaciones sindicales argentinas.

El objetivo principal consistía en crear nuevas herramientas para transparentar y mejorar los modelos de gestión, educación y financiamiento. Poco a poco estas actividades fueron haciéndose estériles en medio de diversas pujas políticas entre la nación, las provincias y los municipios, con el resultado de que muchos de quienes fueron capacitados para dirigir estos cambios fueron reemplazados por otros funcionarios afines a las administraciones del momento. PRESSAL concluyó en 2000 en medio de denuncias de corrupción(4). Dado que estas acusaciones no fueron investigadas (el caso puntual que referimos no generó ningún proceso judicial), no puede aventurarse una conclusión sobre la exactitud de estas denuncias, pero los magros resultados que dejó esta iniciativa permiten aseverar que el esfuerzo fue estéril.

Las intrigas y el feudalismo presentes en el sector público de la salud de Argentina fueron narradas por un sanitarista, Ignacio Katz, quien a fines de los 90 fue designado interventor en un hospital del Gran Buenos Aires -centro de referencia obligado para aproximadamente cuatro millones de personas que pertenecen, en su gran mayoría, a sectores con escasa capacidad económica- por el Ministerio de Salud argentino.

En su libro, "Argentina Hospital”, Katz describe las peripecias sufridas en su intento por ordenar la institución transparentando las actividades de los servicios de compras, farmacia y mantenimiento, además de la forma en que se llevaban a cabo los ensayos clínicos(5).

Tanto lo sucedido con PRESSAL como lo relatado en último caso ofrecen puntos en común: disociación entre la realidad y lo declamado por los sectores políticos y gremiales respecto de la defensa de los derechos de pacientes, y una retórica constante por parte de los organismos responsables, los cuales suelen asociar la crisis existente con meras deficiencias de financiamiento.

Resulta habitual leer explicaciones acerca de la necesidad de establecer principios estratégicos de equidad, universalidad, intersectorialidad y participación social. No es habitual, en cambio, leer referencias sobre la 
responsabilidad que tendría la corrupción en la degradación de los servicios de salud argentinos.

Si bien es imposible medir con exactitud de qué manera la corrupción afecta la calidad y la gestión de los sistemas de salud, debe destacarse que, de acuerdo con Transparencia Internacional, agencia no gubernamental que mide los índices de corrupción percibida en forma anual, para 2009 Argentina se encontraba en la posición 2.9 en el ranking mundial de corrupción percibida (siendo 9.4 el índice para el país con menor índice de corrupción percibida y 1.1 el mayor)(6). Va de suyo que, sea cual fuere la influencia de la escasa transparencia en la administración de los recursos para la salud, es un factor que no debería soslayarse si lo que se pretende es generar una discusión seria sobre los problemas generados por la falta de justicia y equidad de los sistemas de salud públicos y dependientes de la seguridad social, sostenidos financieramente por los impuestos y las contribuciones laborales de la población.

Los problemas de gobernanza que agobian a Argentina inevitablemente se expanden hacia todas las ramas de la actividad pública y privada, modificando paradigmas y estableciendo conductas que permiten la sobrevivencia del más "apto". En el caso de los servicios de salud, estos problemas se ven magnificados por la escasa importancia que las autoridades suelen asignarles a los trabajadores de estas organizaciones (a pesar de los discursos retóricos e inflamados que desgranan ideas de justicia y equidad). Las magras retribuciones que reciben los agentes de la salud potencian el desincentivo de trabajar en el sistema estatal o de la seguridad social. De esta manera, la palabra "calidad" queda vaciada de contenido, sólo útil como muletilla para los discursos y las planificaciones. La frustración y la decepción suele preceder al desinterés y al cinismo. De tal manera, ética y calidad se convierten en conceptos descontextualizados y el pesimismo se extiende a los usuarios, pacientes y comunidad en general.

Calidad, ética y salud navegan entonces no como la resultante de una serie de procesos que pueden ser incorporados por los miembros de la organización, sino como un producto que sólo ocurre en "otra parte". Esa "otra parte" puede estar representada por algunos centros privados del mismo país o por instituciones que funcionan en los llamados "países desarrollados". En cualquier caso, la "otra parte" es ajena. Esto no es óbice para que, de tanto en tanto, en alguno de estos establecimientos se produzca una "proeza médica", y como tal suele ser reflejada en los medios y en la conciencia de la población. Numerosos éxitos en el sistema público de salud argentino son el resultado de esfuerzos y sacrificios de profesionales y auxiliares de la salud quienes, en forma personal, intentan superar las carencias que los abruman. Son los individuos quienes se sobreponen a los límites que impone la organización, pero no es el sistema el que incentiva a los individuos. Al contrario, en muchos casos la organización desquiciada atropella a las individualidades como un autómata ciego, sordo e indiferente.

\section{Abulia ética}

Aunque los casos narrados en este artículo están relacionados con Argentina, es posible extrapolar estas situaciones y sus consecuencias a gran parte de América Latina. Las mejoras en los procesos de la salud pública pueden verse representadas en una ecuación en la cual los resultados positivos dependen de la calidad institucional o gobernanza $(\mathrm{G})$, trabajo realizado $(\mathrm{L})$ y capital utilizado (C). Los incrementos en $\mathrm{L}$ (mayor número de personas en general, mayor productividad) y en $\mathrm{C}$ (infraestructura, equipos médicos y otros bienes durables) pueden tener impactos transitorios, pero la gobernanza $(\mathrm{G})$ influye de manera constante en el funcionamiento de las otras variables(7).

Si se acepta que los fenómenos de corrupción dentro de una sociedad generan inmensas desigualdades en la provisión de servicios de salud, no sería ilógico presumir que la ética, como parte inevitable de la bioética, debería contribuir a una racionalidad emancipadora de esta problemática en vez de legitimar el statu quo(8).

Los 90 fueron prolíficos en replanteos sobre políticas en salud, necesidades de los usuarios y estrategias para responder a la demanda, en tanto se diseńaban intervenciones en salud pública que permitieran disminuir la carga de enfermedad de una sociedad. Estas discusiones fueron el tema dominante en los países desarrollados. Durante la administración del presidente Clinton, Estados Unidos se envolvió en un áspero debate sobre las características de su modelo sanitario. Si bien estas discusiones tuvieron múltiples aristas, sus puntos cruciales se centraron en asegurar la universalidad del acceso al sistema y las medidas para actuar sobre los costos insaciables del modelo vigente. Un poco antes, el Estado de Oregon inició su propio experimento, en el afán por proporcionar servicios básicos de salud a sus 
habitantes no asegurados, así como a aquellos que no calificaban para los planes federales de salud(9).

En la misma década, con un escenario muy distinto al de Estados Unidos, el Reino Unido inició el más ambicioso plan de reformas desde 1948, cuando fue creado el Servicio Nacional de Salud Británico. (NHS)(10). Los países europeos, Canadá y Oceanía no escaparon a la necesidad de establecer prioridades y políticas de financiamiento, en la búsqueda de mayor justicia dentro de un sistema que permitiera la máxima calidad posible.

Más allá de los éxitos o fracasos en las reformas emprendidas, en cada país la comunidad bioética participó activamente en las discusiones sobre los modelos propuestos y el impacto moral que significaban los cambios emprendidos. Con sólo incorporar las palabras clave adecuadas, cualquier buscador en Internet permitirá encontrar centenas de documentos escritos al respecto.

Sería injusto decir que, en América Latina, los cambios propuestos y parcialmente ejecutados en los 90 no tuvieron respuestas por parte de la bioética. Esto, claro, no garantizó que las mismas fueran reflexivas y razonadas.

La bioética latinoamericana comenzó a rebelarse ante lo que percibía como una imposición ética proveniente de los países desarrollados, aunque en general, con origen en Estados Unidos de América, producto, según lo entendían, de una visión mercantilista e individualista, despreocupada de los derechos humanos y de las desigualdades sociales, e indiferente a las necesidades de justicia social y solidaridad. En los casos más extremos, la naturaleza dialógica de la bioética fue reemplazada por una mera repetición de consignas(11).

Esta bioética "comprometida" y "militante" sostiene la necesidad de generar otro modelo que permita luchar contra las inequidades, enarbolando como banderas la defensa de aquellos que sufren por el avasallamiento de sus derechos humanos. Esta bioética, "hace sonar correctamente la alarma sobre la situación social de la región, pero el tratamiento propuesto no encaja en el diagnóstico"(12).

De acuerdo con su visión, la bioética de la "liberación" explica las desigualdades del presente como consecuencia de la historia de explotación y sojuzgamiento de las naciones de América Latina, y no tiene empacho en remontarse hasta la época de la conquista española, entendiendo que la historia es tan estática como una fotografía. El atraso social de las naciones se justificaría por un determinismo inexorable, en una constante lucha entre el bien y mal, estando el bien representado por las clases marginales y sojuzgadas y el mal por quienes, desde dentro y fuera del país, pugnan por perpetuar esta marginación. Ya que los enemigos son históricos y atemporales, parece no ser necesario (por parte de esta bioética) preguntarse si cabe alguna responsabilidad en la perpetuación de las injusticias a la escasa transparencia en el uso de los recursos por parte de los responsables de los sistemas de salud estatales, provinciales, municipales y de la seguridad social, así como a las dificultades de gobernanza extendidas por todo el tejido social.

Frente a la indiferencia de la bioética tradicional -ocupada en los problemas "clásicos" - y la vocinglería de la bioética "dura", el desenlace institucional y social resulta ser una especie de "atonía ética", generada por el acostumbramiento ante la reiteración de los mismos estímulos. Ante la falta de alternativas, la adaptación es la única conducta que puede permitir la sobrevivencia.

En tanto las discusiones se centren en aspectos universales - totalmente inasibles—, no es de extrañar que la bioética no encuentre aún su lugar en los razonamientos sobre las reformas necesarias para que los procesos de calidad y de eficiencia puedan finalmente impactar sobre quienes realmente necesitan un cambio en los paradigmas de atención médica, representados por la inmensa mayoría de habitantes cuya única opción es recibir cuidados sanitarios brindados por el sistema público o la seguridad social.

\section{Conclusiones}

En Argentina (así como en muchas otras naciones de América Latina) las metas de salud para todos se plantearon mediante la búsqueda de criterios de equidad, solidaridad, eficacia, eficiencia y calidad. Se esperaba incorporar recursos normativos, organizativos, instrumentales, de gestión y fiscalización de los distintos aspectos relacionados con el proceso global de atención médica(13). No obstante estos enunciados ampulosos, poco se ha hecho por instrumentar medidas que permitieran mejorar los indicadores de calidad. Por el contrario, en muchos casos el fragmentado sistema de salud público argentino desperdicia recursos al subsi- 
diar al sector de la seguridad social, administrado por el sector sindical, ya que muchos derechohabientes del mismo terminan recibiendo atención médica en el sector público sin que haya reembolso por los gastos ocasionados. La escasa transparencia -patognomónica en los procesos de contratación de servicios o compra de bienes- afecta en forma directa a quienes contribuyen al financiamiento del mismo mediante impuestos y aportes de trabajadores y empleadores. Un escaso porcentaje de la población tiene una tercera imposición, al pagar de su bolsillo un seguro privado de salud.

Algunas voces de la bioética local entienden que los culpables de este estado de cosas, cuya resultante son injusticias e inequidades en el terreno de salud, son intereses ajenos al país, en complicidad con "elites nacionales dominantes" (14). Esta visión reduccionista, compartida por algunos colegas brasileños, pone de manifiesto una justificación paternalista, ya que atribuye la carencia de bienes básicos primarios, como el acceso a la salud o la mala calidad de los servicios ofrecidos, a la aplicación de políticas y filosofías provenientes de los países desarrollados, los cuales tendrían un interés especial en colonizar (una vez más) a los llamados "en vías de desarrollo". Así, los modelos de exclusión resultantes son la consecuencia de actos externos en cuyas consecuencias los agentes locales tendrían escasa responsabilidad.

Resulta llamativo que, más allá de pedidos de justicia y de respeto a los derechos humanos, los cuales - por abstractos y universales- se convierten en enunciados retóricos, los problemas derivados de la corrupción y la falta de transparencia en el sector de la salud no mere- cen expresiones de similar contundencia. Al contrario, al omitir las consecuencias de la falta de gobernanza como una de las causas principales de las inequidades en salud, estas invocaciones resultan tranquilizadoras, por cuanto los principios enunciados responden a consignas consideradas políticamente progresistas que, aunque estériles, permiten excitantes ejercicios dialécticos que no requieren de rigurosidad analítica, al no poder ser confrontados con acciones capaces de modificar el estado de las cosas.

Si la bioética como disciplina puede ser considerada como una herramienta más en el proceso de la toma de decisiones en relación con la dinámica y funcionamiento de los sistemas de salud, es de esperar que en algún momento pueda ser partícipe en el enfoque de tópicos tan complejos como el mejorar la accesibilidad y la equidad de éstos.

En países que, como Argentina, tienen una larga tradición de intolerancia y problemas serios de gobernanza resulta indispensable fortalecer el ejercicio del pensamiento crítico en las discusiones sobre el mejor uso de los recursos públicos para la prevención y tratamiento de la enfermedad. Ese tal vez sea el mejor aporte para que las ideas enunciadas hace casi tres décadas en Alma Ata se articulen con los principios de respeto, beneficencia y justicia, disminuyendo las disparidades y asegurando que salud para todos pueda ser, más allá de un bonito enunciado, algo tangible para los millones de excluidos cuya única esperanza, aunque no lo sepan, es contar con un modelo de salud más transparente, dentro de una sociedad con nula tolerancia a los actos de corrupción.

\section{Referencias}

1. UNICEF-WHO. Declaration of Alma Ata International Conference on Primary Health Care. Alma-Ata, URRS, 6-12 September, 1978. Disponible en http://www.who.int/hpr/NPH/docs/declaration_almaata.pdf

2. Donabedian A. Evaluating the quality of medical care. Millbank Fund Quarterly 1982; 44(2): 166-206.

3. Rodríguez Yunta E. Temas para una bioética latinoamericana. Acta Bioethica 2009;15(1): 87-93.

4. Argentina, Oficina Anticorrupción, Ministerio de Justicia de la República. Relato de irregularidades identificadas en: Programa de Reformas del Sector Salud (PRESSAL), 2000. Disponible en: http://www.anticorrupcion.gov.ar/Varias\%20 irregularidades\%20en\%20PRESSAL.pdf

5. Katz I. Argentina Hospital. El rostro oscuro de la salud. Buenos Aires: Edhassa; 2004.

6. Transparencia Internacional. Índice de Corrupción Percibida, 2009. Disponible en: http://www.transparency.org/ policy_research/surveys_indices/cpi/2009/cpi_2009_table

7. Lewis M. Governance and Corruption in Public Health Systems. Working Paper 78. Center for Global Development; 2008. 
Calidad, sistema de salud y gobernanza. El caso argentino - Sergio G. Litewka

8. Outomuro D. Algunas observaciones sobre el estado actual de la bioética en Argentina. Acta Bioethica 2003; 9(2): 229-238.

9. Saha S, Coffman DD, Smits AK. Giving teeth to comparative-effectiveness research. The Oregon experience. N Engl J Med. 2010; 362(7): e18.

10. Best G, Knowles D, Mathew D. Managing the new NHS: breathing new life into the NHS reforms Gordon Best. British Medical Journal 1994; (308): 842-845.

11. Legarda C. Una lectura crítica de la bioética latinoamericana. En: Pessini L, De Barchifontaine ChdeP, Lolas F. (coord.) Perspectivas de la Bioética en América Latina. Santiago de Chile: Programa de Bioética OPS/OMS; 2007: 249-260.

12. Rodríguez del Pozo P, Mainetti J. Bioética sin más. Present, past and future of a Latin American Bioethics. Cambridge Quarterly of Healthcare Ethics 2009; 270: 279.

13. Ministerio de Salud y Acción Social de la Nación. La garantía de calidad en el hospital público de autogestión: Marco conceptual, estrategias e instrumentos operativos. Buenos Aires: Editorial Médica Panamericana; 1997.

14. Justo L. La ética de la investigación social en América Latina: del consentimiento informado a la participación crítica. Conferencia. Primer simposio para comités de ética en investigación en Centroamérica. Costa Rica 27, 282003 Disponible en http://e-ms.cilea.it/archive/00000548/01/Justo_-_La_\%C3\%A9tica_de_la_investigaci\%C3\%B3n_ cl\%C3\%ADnica_-_Del_consentimiento_informado_a_la_participaci\%C3\%B3n_cr\%C3\%ADtica.pdf

Recibido: 23 de junio de 2010

Aceptado: 15 de julio de 2010 